\title{
Focal Segmental Glomerulosclerosis and Recurrence in Living Donor Recipients
}

\author{
Josef Mang' \\ Linda Hennig' \\ lutz Liefeldt ${ }^{2}$ \\ Michael Duerr ${ }^{2}$ \\ Lukas J Lehner ${ }^{2}$ \\ Anna Bichmann (iD) 3 \\ Bernhard Ralla' \\ Hannes Cash ${ }^{4}$ \\ Martin Christopher Kanne ${ }^{5}$ \\ Robert Peters' \\ Andreas Maxeiner ${ }^{1} *$ \\ Frank Friedersdorff ${ }^{1,5}, *$ \\ 'Department of Urology, Charité - \\ Universitätsmedizin Berlin, Corporate \\ Member of Freie Universität Berlin, \\ Humboldt-Universität zu Berlin, and \\ Berlin Institute of Health, Berlin, \\ Germany; ${ }^{2}$ Department of Nephrology, \\ Charité - Universitätsmedizin Berlin, \\ Corporate Member of Freie Universität \\ Berlin, Humboldt-Universität zu Berlin, \\ and Berlin Institute of Health, Berlin, \\ Germany; ${ }^{3}$ Department of \\ Anesthesiology and Operative Intensive \\ Care Medicine, Campus Charité Mitte, \\ Charité - Universitätsmedizin Berlin, \\ Corporate Member of Freie Universität \\ Berlin, Humboldt-Universität zu Berlin, \\ and Berlin Institute of Health, Berlin, \\ Germany; ${ }^{4}$ PROURO, Berlin, Germany; \\ ${ }^{5}$ Department of Urology, Evangelisches \\ Krankenhaus Königin Elisabeth \\ Herzberge, Berlin, Germany \\ *These authors contributed equally to \\ this work
}

Correspondence: Linda Hennig Department of Urology, Charité Universitätsmedizin Berlin, Corporate

Member of Freie Universität Berlin,

Humboldt-Universität zu Berlin, and

Berlin Institute of Health, Charitéplatz I,

Berlin, I0117, Germany

Email linda.hennig@charite.de
Purpose: Focal segmental glomerulosclerosis (FSGS) is a common cause for end-stage renal disease that can recur in the graft after kidney transplantation. The incidence of FSGS recurrence is reported in up to $47 \%$ of patients, predisposing those to possible poorer transplantation outcomes. Hence, we examined the incidence of FSGS recurrence and the effect on graft outcome in our patient cohort of living donor kidney transplantations (LDKT). Patients and Methods: We analyzed 194 adult patients who received a LDKT between 2011 and 2017 of which 22 (11\%) had FSGS as underlying disease. Demographic data and clinical outcomes, especially regarding recurrence of FSGS, were evaluated.

Results: FSGS recurrence was identified in three (14\%) patients within three months after transplantation, of whom two patients $(9 \%)$ lost their graft. There was no significant difference in graft survival comparing FSGS to other reasons for end-stage renal disease.

Conclusion: Incidence of FSGS recurrence in the present patient cohort was within the range reported in the literature and comparatively low. Our data support LDKT as a treatment option in patients with end-stage renal disease due to FSGS.

Keywords: FSGS, living donor nephrectomy, recurrence

\section{Introduction}

Focal segmental glomerulosclerosis (FSGS) is a common cause for end-stage renal disease (ESRD) and in the absence of an underlying cause leading to FSGS is defined as primary FSGS. ${ }^{1}$ Diagnosis of primary FSGS is of particular importance for patients being evaluated for kidney transplantation, since FSGS can recur in the kidney transplant with a risk of consecutive graft loss. ${ }^{2}$ Recurrence rates in literature vary between $10.3 \%$ and $47 \%$ and graft survival is reported to be shorter in patients with FSGS compared with other reasons leading to end-stage renal disease. ${ }^{3-10}$

The risk of FSGS recurrence has to be considered in the evaluation of patients for living donor kidney transplantation (LDKT) since the positive effects for the recipient also come with risks for the donor. There is conflicting evidence regarding the outcome for LDKT in patients with FSGS. While some data suggest no advantage in LDKT compared with deceased donor transplantation, other authors see an advantage for LDKT on graft survival in patients suffering from FSGS., ${ }^{3,11}$

However, kidney transplantation, either living or deceased donor based, is considered to be the best treatment option for ESRD in patients with FSGS.

We therefore (I) analyzed the incidence of FSGS recurrence and (II) the effect on graft outcome in a single high-volume transplantation center for patients with FSGS who received LDKT. 


\section{Materials and Methods}

\section{Patients}

For the present study all adult patients who received LDKT between 2011 and 2017 at Charité Hospital Berlin, Department for Urology, were analyzed for the underlying cause of end-stage renal disease. Patients with suspicion of primary FSGS were included in this study. Diagnosis of FSGS was made by biopsy based on histological findings and electron microscopy. Patients were screened for virus associated, structural anomalies or ischemic reasons as underlying cause of FSGS. However, genetic testing was not performed routinely, but was performed in patients with family history of FSGS. In the absence of a reason for secondary FSGS patients were considered to be suffering from primary FSGS. Patient demographics, laboratory parameters and graft survival data including pathological and clinical information on FSGS recurrence was extracted from their medical reports. In patients with proteinuria or nephrotic syndrome in suspicion for FSGS recurrence the diagnoses were based on the biopsy and histological examination results.

Patient preparation and surgical technique (laparoscopic donor nephrectomy and renal transplantation) were described in a previous study. ${ }^{12}$

\section{Statistical Analysis}

Statistical analysis of the data was performed using both Microsoft Office Excel 2007 and IBM SPSS Statistics 25 software. Statistical analysis for Table 1 was done by $t$-test. Kaplan-Meier analysis was done to compare graft survival data (Log rank test), $p$-values $<0.05$ were considered statistically significant.

\section{Results}

Between 2011 and 2017 LDKT were performed in 194 adults at our center. FSGS was the reason for end-stage renal disease in 22 patients (11\%). Comparison of patient characteristics are shown in Table 1. Recipients composed of 13 males and 9 females, mean age ( 37 years) and BMI $\left(23.7 \mathrm{~kg} / \mathrm{m}^{2}\right)$ were significantly lower in patients who underwent LDKT with FSGS compared with the rest of our collective (46.1 years and $25.9 \mathrm{~kg} / \mathrm{m}^{2}$ respectively). There was no significant difference in pre- and postoperative serum creatinine levels presented in Table 1 with a mean follow up of 46.7 and 44.3 months respectively.

Out of 22 patients, three patients (14\%) developed recurrence of FSGS (Table 2) of whom two patients
Table I Comparison of Patient Characteristics

\begin{tabular}{|l|c|c|c|}
\hline & FSGS & No FSGS & p-value \\
\hline $\mathrm{n}$ & 22 & 172 & \\
Age (years) & 37 & 46.1 & 0.007 \\
BMI (kg/m $\left.{ }^{2}\right)$ & 23.7 & 25.9 & 0.008 \\
\hline Serum Creatinine (mg/dl) & & & \\
Pre-surgery & 7.6 & 7.1 & n.s. \\
$\quad$ I week p. s. & 2.9 & 2.5 & n.s. \\
I month p. s. & 1.7 & 1.6 & n.s. \\
3 months p. s. & 1.6 & 1.6 & n.s. \\
6 months p. s. & 1.7 & 1.5 & n.s. \\
I year p. s. & 1.9 & 1.4 & n.s. \\
2 years p. s. & 1.3 & 1.5 & n.s. \\
3 years p. s. & 1.3 & 1.5 & n.s. \\
4 years p. s. & 1.5 & 1.5 & n.s. \\
5 years p. s. & 1.4 & 1.6 & n.s. \\
\hline Follow-up time (months) & 46.7 & 44.3 & n.s. \\
\hline Waiting time (m) & 15 & 14 & n.s. \\
\hline ABO-incompatible & $3(14 \%)$ & $5 \mathrm{I}(30 \%)$ & \\
\hline Rejection & $4(18 \%)$ & $26(15 \%)$ & \\
\hline
\end{tabular}

Abbreviations: BMI, body mass index; p.s., post surgery.

(9\%) lost their graft due to FSGS recurrence. In all cases, FSGS recurrence was diagnosed within three months post-transplantation. The treatment in those patients was initiated instantly, yet one patient lost the graft within months after transplantation, whereas the second patient lost his graft after 93 months. The third patient suffering from FSGS recurrence still shows good kidney function with now 89 months of follow up.

In this patient cohort one other graft loss occurred for other reason than FSGS recurrence with no primary graft function.

Kaplan-Meier analysis on graft survival in the FSGS group compared with all patients who underwent LDKT showed no significant difference $(p=0.37)$ on graft survival as shown in Figure 1.

\section{Discussion}

The data published on incidence of FSGS recurrence after kidney transplantation varies widely. A potential reason could be that many studies were based on small patient size and thereby under- or overestimate the real incidence of recurrence. Furthermore, FSGS recurrence is only seen in patients with primary FSGS, which can only be diagnosed after the exclusion of all other causes for secondary 


\begin{tabular}{|c|c|c|c|}
\hline 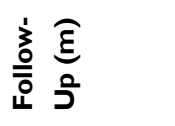 & ఫे & ळ & $\alpha$ \\
\hline 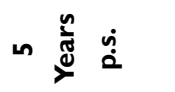 & 1 & $\stackrel{\stackrel{n}{N}}{\longrightarrow}$ & $\stackrel{m}{i}$ \\
\hline 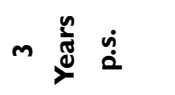 & 1 & $\stackrel{ \pm}{=}$ & $\bar{a}$ \\
\hline - 离 & $\begin{array}{l}\tilde{\sigma} \\
\underline{\underline{m}}\end{array}$ & 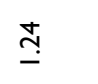 & $\hat{0}$ \\
\hline ○旁 & $\stackrel{n}{r}$ & o̊ & $\stackrel{\mathbb{Z}}{\underline{Z}}$ \\
\hline 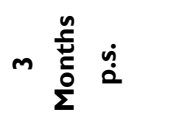 & $\frac{\circ}{\dot{\sigma}}$ & $\stackrel{m}{\underline{q}}$ & $\underline{m}$ \\
\hline 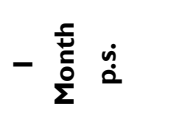 & io & $\stackrel{\underset{I}{I}}{=}$ & $\stackrel{2}{\stackrel{2}{\longrightarrow}}$ \\
\hline 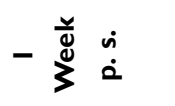 & $\underset{\infty}{\mathcal{Z}}$ & 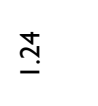 & $\underline{a}$ \\
\hline 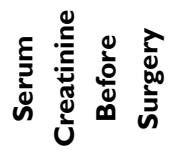 & 脶 & $\underset{j}{\sigma}$ & $\stackrel{T}{\sim}$ \\
\hline 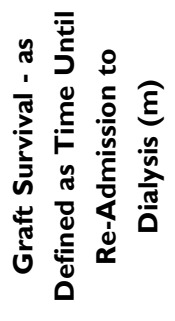 & $\stackrel{\circ}{i}$ & ळ & $\stackrel{\infty}{\sigma_{\alpha}}$ \\
\hline 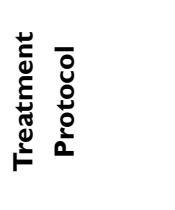 & 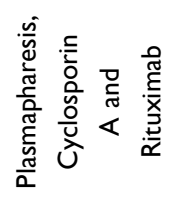 & 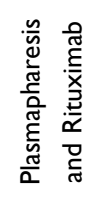 & 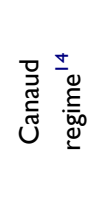 \\
\hline 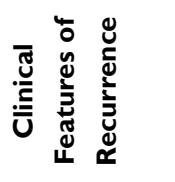 & 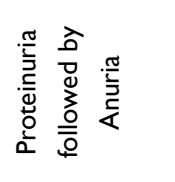 & 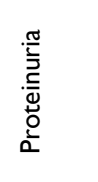 & 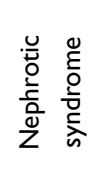 \\
\hline 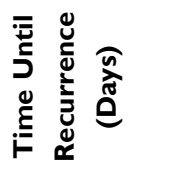 & $N$ & $\bar{\sigma}$ & $\tilde{0}$ \\
\hline ڤ్ & $\Sigma$ & 3 & 3 \\
\hline$\stackrel{8}{\&}$ & $\bar{m}$ & 㶽 & $\approx$ \\
\hline
\end{tabular}

FSGS. ${ }^{1}$ Inclusion of patients with undiagnosed secondary FSGS might thereby lead to smaller recurrence rates. However, in our sample we identified FSGS recurrence in $14 \%$ of patients. Thus, an incidence in the lower range of reported incidences might be potentially explained by our small sample size. Also, there might be different reasons leading to inclusion of secondary FSGS in this study, and thereby being a limitation interpreting the low recurrence rates. Since not every patient is diagnosed with FSGS in our clinic and is evaluated in our center for transplantation, we have to rely on diagnosis and patient history. Furthermore, most patients in this collective did not receive genetic testing since it was not performed routinely.

There are conflicting data on graft survival in patients with FSGS compared with other reasons for ESRD. While Francis et al. showed a significantly worse graft survival rate in patients with FSGS compared with their control group, other authors failed to identify a significant difference in their patient cohorts. ${ }^{3,6,13}$

However, in our patient cohort, there was no significant difference in graft survival in patients with FSGS compared with our control group, potentially due to the low recurrence rates and small sample size. Also, a limitation is, that we cannot provide longer follow up than 80 months in our control group. With one graft loss in the FSGS subgroup after 93 months comparing those groups in longer follow up might have led to a different result.

Despite the varying results on graft survival in all patients suffering from FSGS, there seems to be clear evidence of earlier graft loss in patients who develop FSGS recurrence compared with those without recurrence. ${ }^{3,6,13}$ In the large multicentric TANGO study, Uffing et al. showed an up to 5-fold risk for graft loss for patients who develop recurrence, highlighting the need for a yet to be established standardized treatment protocol for FSGS recurrence. ${ }^{9}$ Also developing treatment protocols to prevent FSGS recurrence in the first place are important tasks for future investigations.

The question whether to evaluate patients with FSGS for LDKT is highly debated.

Available data suggest graft survival from LDKT to be superior compared with deceased donor kidney transplantation. ${ }^{3}$ The results of this study do not contradict the evaluation of patients suffering from FSGS for LDKT. Kidney transplantation still may be the best treatment option for patients with end-stage renal disease suffering from FSGS. 


\section{Kaplan Meier Analysis}

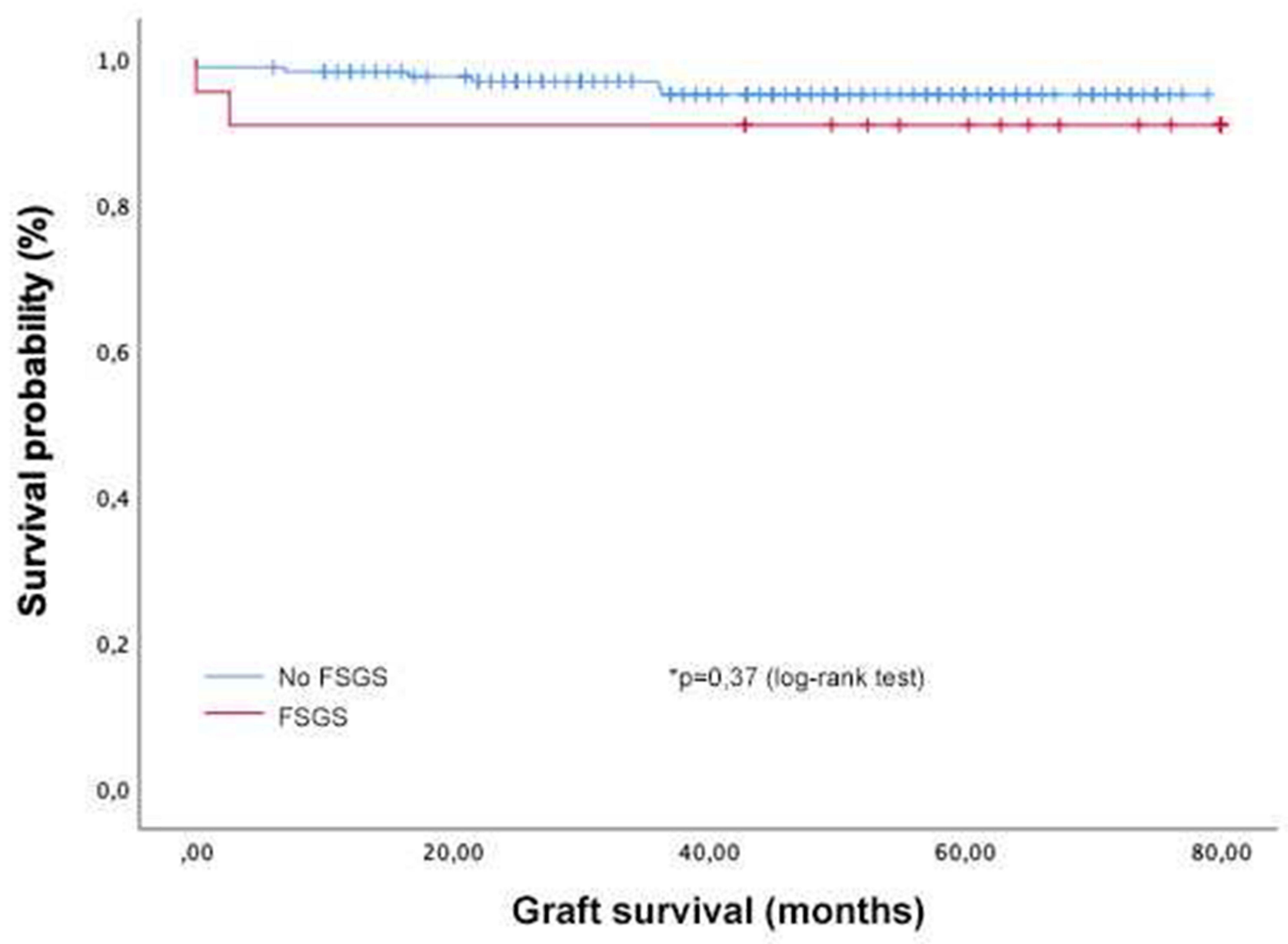

Figure I Kaplan-Meier Analysis.

\section{Statement of Ethics}

The data presented in this study was conducted ethically in accordance with the World Medical Association Declaration of Helsinki. All organs were donated voluntarily with written informed consent, and this was conducted in accordance with the Declaration of Istanbul. For retrospective analysis patients were provided with a waiver with consent information. The study was approved by the ethics committee of Charité - Universitätsmedizin.

\section{Author Contributions}

All authors made substantial contributions to conception and design, acquisition of data, or analysis and interpretation of data; took part in drafting the article or revising it critically for important intellectual content; agreed to submit to the current journal; gave final approval of the version to be published; and agree to be accountable for all aspects of the work. All authors made a significant contribution to the work reported, whether that is in the conception, study design, execution, acquisition of data, analysis and interpretation, or in all these areas; took part in drafting, revising or critically reviewing the article; gave final approval of the version to be published; have agreed on the journal to which the article has been submitted; and agree to be accountable for all aspects of the work.

\section{Disclosure}

Dr Michael Duerr reports personal fees from Novartis Pharma GmbH and Takeda Pharmaceutical Company Limited, outside the submitted work. All authors declare that they have no other competing interests. 


\section{References}

1. Sprangers B, Meijers B, Appel G. FSGS: diagnosis and diagnostic work-up. Biomed Res Int. 2016;2016(2314):4632768. doi:10.1155/ 2016/4632768

2. Kienzl-Wagner K, Waldegger S, Schneeberger S. Disease recurrence-the sword of damocles in kidney transplantation for primary focal segmental glomerulosclerosis. Front Immunol. 2019;10 (1664):1669. doi:10.3389/fimmu.2019.01669

3. Francis A, Trnka P, McTaggart SJ. Long-term outcome of kidney transplantation in recipients with focal segmental glomerulosclerosis. Clin J Am Soc Nephrol. 2016;11(11):2041-2046. doi:10.2215/ CJN.03060316

4. Hickson LJ, Gera M, Amer H, et al. Kidney transplantation for primary focal segmental glomerulosclerosis: outcomes and response to therapy for recurrence. Transplantation. 2009;87(8):1232-1239. doi:10.1097/TP.0b013e31819f12be

5. Maas RJH, Deegens JKJ, van den Brand JAJG, Cornelissen EAM, Wetzels JFM. A retrospective study of focal segmental glomerulosclerosis: clinical criteria can identify patients at high risk for recurrent disease after first renal transplantation. BMC Nephrol. 2013;14(1). doi:10.1186/1471-2369-14-47

6. Moroni G, Gallelli B, Quaglini S, Banfi G, Montagnino G, Messa P. Long-term outcome of renal transplantation in adults with focal segmental glomerulosclerosis. Transplant Int. 2010;23(2):208-216. doi:10.1111/j.1432-2277.2009.00977.x

7. Pardon A, Audard V, Caillard S, et al. Risk factors and outcome of focal and segmental glomerulosclerosis recurrence in adult renal transplant recipients. Nephrol Dial Transplant. 2006;21(4):1053-1059. doi:10.1093/ndt/gfk005
8. Rudnicki M. FSGS recurrence in adults after renal transplantation. Biomed Res Int. 2016;2016(2314):3295618. doi:10.1155/2016/ 3295618

9. Uffing A, Perez-Saez MJ, Mazzali M, et al. Recurrence of FSGS after kidney transplantation in adults. Clin J Am Soc Nephrol. 2020;15 (2):247-256. doi:10.2215/CJN.08970719

10. Cosio FG, Frankel WL, Pelletier RP, Pesavento TE, Henry ML, Ferguson RM. Focal segmental glomerulosclerosis in renal allografts with chronic nephropathy: implications for graft survival. Am J Kidney Dis. 1999;34(4):731-738. doi:10.1016/S0272-6386(99) 70400-2

11. Baum MA, Stablein DM, Panzarino VM, Tejani A, Harmon WE, Alexander SR. Loss of living donor renal allograft survival advantage in children with focal segmental glomerulosclerosis. Kidney Int. 2001;59(1):328-333. doi:10.1046/j.1523-1755.2001.00494.x

12. Mang J, Hennig L, Biernath N, et al. Is a retroaortic vein a risk factor in laparoscopic living donor nephrectomy? Urol Int. 2020;104(78):641-645. doi:10.1159/000507642

13. Staeck O, Halleck F, Budde K, Khadzhynov D. Long-term outcomes of kidney transplant recipients with primary idiopathic focal segmental glomerulosclerosis. Transplant Proc. 2017;49(10):2256-2259. doi:10.1016/j.transproceed.2017.10.001

14. Canaud G, Zuber J, Sberro R, et al. Intensive and prolonged treatment of focal and segmental glomerulosclerosis recurrence in adult kidney transplant recipients: a pilot study. Am J Transplant. 2009;9 (5):1081-1086. doi:10.1111/j.1600-6143.2009.02580.x
Research and Reports in Urology

\section{Publish your work in this journal}

Research and Reports in Urology is an international, peer-reviewed, open access journal publishing original research, reports, editorials, reviews and commentaries on all aspects of adult and pediatric urology in the clinic and laboratory including the following topics: Pathology, pathophysiology of urological disease; Investigation and treatment of urological disease; Pharmacology of drugs used for the treatment of urological disease. The manuscript management system is completely online and includes a very quick and fair peer-review system, which is all easy to use. Visit http://www.dovepress.com/ testimonials.php to read real quotes from published authors. 